\title{
Das Experiment: Lernversuche am Menschen
}

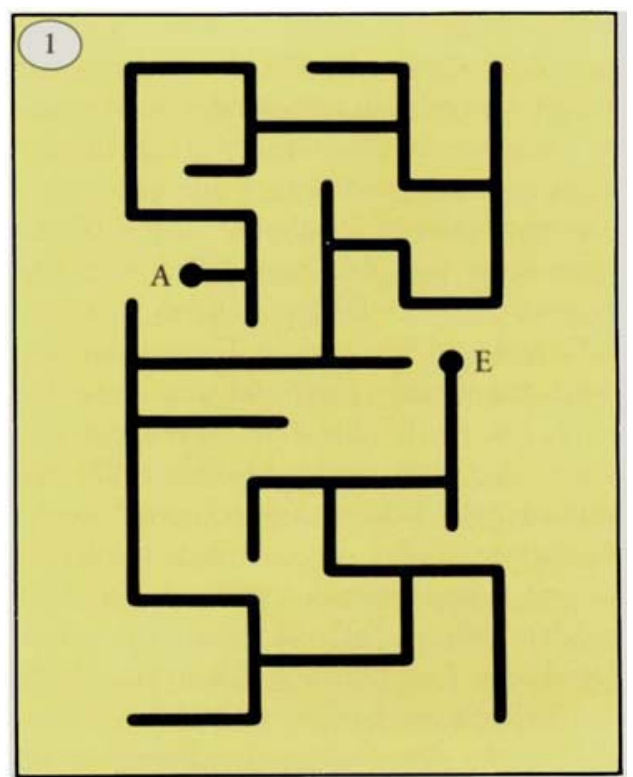

Abb. 1. Beispiel für ein Labyrinth.
Ein wichtiger Bereich der Ethologie, nämlich derjenige, der sich mit der Untersuchung des Lernverbaltens bei Tieren und Menschen befaßt, findet vor allem deshalb nur schwer Eingang in den Schulunterricht, weil einfache Experimente zur Veranschaulichung dieses Stoffes kaum bekannt sind. Im folgenden soll daher ein sowohl vom materiellen als auch vom zeitlichen Aufwand her sehr einfaches Experiment zur quantitativen $\mathrm{Un}_{\mathrm{n}}$ tersuchung eines Lernvorgangs beschrieben werden. Dieses Experiment hat dabei den methodischen wie didaktischen Vorteil, daß es an Menschen durchgeführt werden kann. Ein weiterer Vorteil des Experimentes liegt darin, daß es sich gut dafür eignet, bei der Erarbeitung des Versuchsplanes die Fähigkeit zu fördern, methodische Ansätze kritisch beurteilen sowie unterschiedliche Methoden gegeneinander abwägen zu können.

Die apparativen Voraussetzungen sind gering: Neben einer Stoppuhr, Bleistift und Papier wird eine Augenbinde benötigt, die aber auch, für die Versuchsperson bequemer, durch eine gänzlich schwarz lackierte Laborbrille ersetzt werden kann. Das Kernstück der Versuchsanordnung bildet ein Labyrinth, das aus einer Metall-, Kunststoff- oder Sperrholzplatte (je nach Material 2-5 mm dick) leicht selbst hergestellt werden kann. In diese Platte werden als Anfangs- und Endpunkt des Labyrinthes zwei Löcher gebohrt (Durchmesser 5-10 mm), die durch ausgesägte Schlitze (Breite 4-5 mm) miteinander verbunden sind. In Abbildung 1 ist als Beispiel ein derartiges Labyrinth dargestellt. (Durch Umdrehen der Platte erhält man ein zweites, zum ersten spiegelbildliches Labyrinth). Neben der Versuchsperson wird noch eine zweite Person als Protokollführer benötigt.

Die Aufgabe der Versuchsperson bei der Ausführung des Experimentes besteht darin, mit verbundenen Augen das vor ihr flach auf dem Tisch liegende Labyrinth mit einem Bleistift möglichst fehlerfrei, das heißt, auf dem kürzesten Wege vom Anfangspunkt bis zum Endpunkt zu durchfahren. Zu Beginn jedes neuen Durchgangs setzt der Protokollführer die Bleistiftspitze der Versuchsperson wieder in den Startpunkt. (Unter das Labyrinth kann ein Blatt Papier gelegt werden, wenn man den Weg der Versuchsperson zu Demonstrationszwecken aufzeichnen will).

Vor dem Versuchsbeginn muß die Frage diskutiert werden, auf welche Weise der
Lernerfolg quantitativ erfaßt werden soll. Drei einfache Möglichkeiten kommen in Frage:

1. Es werden für jeden Durchgang die Zabl der Febler $F$ registriert, die dadurch gemacht werden, daß die Versuchsperson an einer Verzweigungsstelle des Labyrinthes in einen blinden Ast gerät. Ebenso könnte man natürlich die Zahl der richtigen Entscheidungen bestimmen.

2. Es wird mit Hilfe einer Stoppuhr die Zeit gemessen, die für jeden Durchgang gebraucht wird.

3. Beide Meßmethoden, Zählen der Fehler sowie Messen der Zeit, werden gleichzeitig für jeden Durchgang angewandt.

Sind diese drei Meßmethoden gleichwertig oder gibt es Unterschiede? Man könnte vermuten, daß mit Methode 1 etwas anderes als mit Methode 2 und 3 gemessen wird, da sich die Versuchsperson auf Grund der verschiedenen Methoden in unterschiedlichen psychologischen Zuständen befinden könnte. Bei der ersten Methode wird nämlich von der Versuchsperson nur verlangt, möglichst wenige Fehler zu machen, wobei sie die Geschwindigkeit völlig frei wählen kann, während die Versuchsperson bei der zweiten und dritten Methode außerdem noch unter Zeitdruck gesetzt wird. Man kann daher nicht von vorneherein sagen, ob diese zusätzliche Belastung etwa den Lernerfolg verringert oder zum Beispiel über einen Zwang zu höherer Konzentration vielleicht erhöht. Aber auch wenn dieser Unterschied keine Rolle spielen sollte, sind Methode 1 und Methode 2 nicht miteinander vergleichbar, da mit den beiden Methoden ja ganz verschiedene Meßgrößen erfaßt werden, die auch unter sonst gleichen Voraussetzungen einen unterschiedlichen Zeitverlauf des Lernvorganges zeigen könnten.

Der durch die verschiedenen Meßmethoden hervorgerufene Unterschied läßt sich einfach mit Hilfe der Methode 3 bestimmen, da hier beide Meßmethoden (Auszählen der Fehler und Messen der Zeit) unter sonst konstanten Bedingungen getestet werden. Die Ergebnisse eines auf diese Weise durchgeführten Experimentes sind in Abbildung 2 angegeben. Die Meßwerte werden in Form einer sogenannten Lernkurve dargestellt, indem auf $\operatorname{der} \mathrm{Ab}$ szisse die Anzahl der fortlaufend numerierten Durchgänge, auf der Ordinate die Meßgröße 
(Zahl der falschen Entscheidungen $F$ oder gemessene Zeit) aufgetragen werden. Die Ergebnisse für die Messung der Fehlerzahl sind als Punkte, die für die Messung der Zeit als Kreuze eingezeichnet. (Die Zeit für die Durchführung der in Abbildung 2 dargestellten Messungen, die von Studenten des tierphysiologischen Großpraktikums der Universität Kaiserslautern durchgeführt wurden, betrug etwa 30 min.) Die Lernkurven gliedern sich nach dieser Darstellungsweise in einen ansteigenden und in einen horizontalen Ast. Der vom ansteigenden Ast bezeichnete Bereich wird Lemphase, der vom horizontalen Ast bezeichnete Bereich Kannphase oder Lernplateau genannt. Nach Erreichen des Lernplateaus tritt keine meßbare Verbesserung der Lernleistung mehr auf. Abbildung 2 zeigt, wie sehr eine Aussage über die Beendigung der Lernphase von der Wahl der Meßmethode abhängen kann, da das Lernplateau gemessen durch das Auszählen der Fehler schon etwa nach dem 10. Durchgang, gemessen nach der Zeit aber erst etwa nach dem 25. Durchgang oder noch später erreicht wird. Man muß daher aus diesem Ergebnis den Schluß ziehen, daß, wie oben vermutet, beide Meßmethoden keineswegs vergleichbar sind, sondern daß offenbar mit beiden Methoden zwei verschiedene Lernvorgänge gemessen werden. Eine mögliche Deutung wäre die, daß mit der Messung der Fehlerzahl das Lernen der Form des Labyrinthes, also der Abspeicherung seiner geometrischen Form im Gedächtnis, gemessen wird, während bei der Messung der Zeit außerdem noch ein motorisches Lernen gemessen wird. Damit ist gemeint, daß während dieses Lernvorganges das genaue Steuer- programm für die entsprechende Muskulatur noch verbessert wird. Dieser Prozeß würde nach dieser Vermutung noch andauern, nachdem das Lernen der geometrischen Form des Labyrinthes schon abgeschlossen ist. Eine andere Deutungsmöglichkeit wäre beispielsweise die, daß mit der Methode des Auszählens der Fehler nur die Reihenfolge der nacheinander notwendigen Rechts-LinksEntscheidungen, mit der Methode der Zeitmessung noch zusätzlich das Lernen der Abstände zwischen den einzelnen Verzweigungen gemessen wird. Beherrscht man nämlich die richtige Reihenfolge, so macht man zwar keine Fehler mehr, braucht aber zum Auffinden der Verzweigungsstellen längere Zeit, als wenn der Abstand ebenfalls bekannt ist.

$\mathrm{Zu}$ diesen und eventuell anderen Deutungsmöglichkeiten können weitere Experimente ausgedacht und durchgeführt werden. Im folgenden soll noch diskutiert werden, wie die oben ausgesprochene Vermutung, daß der durch die Meßmethoden 2 und 3 im Gegensatz zu Meßmethode 1 zusätzlich erzeugte Zeitdruck einen Einfluß auf den Lernerfolg ausüben könnte, untersucht werden kann. Dies darf nicht durch einen Vergleich der Ergebnisse der Methode 1 mit denen der Methode 2 geschehen. Mit beiden Methoden werden nämlich, wie bereits festgestellt, verschiedene Vorgänge gemessen, die auch unter sonst gleichen Voraussetzungen einen unterschiedlichen Zeitverlauf zeigen. Eine Untersuchung des Einflusses des zusätzlichen Zeitdruckes ist aber möglich, wenn die Ergebnisse der Methode 1, also bei Messung der Zahl der Fehler ohne Zeit- druck, verglichen werden mit der Messung der Zahl der unter Zeitdruck erreichten Fehler, also mit den nach der dritten Methode gewonnenen Ergebnissen. Möglich ist aber auch, jedesmal gleichzeitig beide Meßmethoden anzuwenden (Methode 3), einmal der Versuchsperson aber mitzuteilen, daß eine möglichst geringe Fehlerzahl erreicht werden soll, während im zweiten Experiment eine möglichst geringe Durchgangszeit gefordert wird. Ein Einfluß des Zeitdruckes muß sich dann in einem unterschiedlichen Verlauf entweder der beiden "Fehlerkurven" beider Experimente oder der beiden "Zeitkurven“ beider Experimente auswirken. Der Vergleich zweier Lernkurven kann sowohl an Hand der Lerngeschwindigkeit (das heißt der Steilheit der Lernkurve in der Anfangsphase) oder der Zeit bis zum Erreichen des Plateaus einerseits wie auch an Hand der je nach Versuchsbedingungen erreichten Höbe des Plateaus geschehen. Mißt man sowohl "Fehlerkurve" als auch „Zeitkurve“, und ändern sich beide in beiden Experimenten nicht gleichsinnig, so bedeutet dies, daß der Zeitdruck auf die beiden mit den verschiedenen Meßmethoden erfaßten Lernvorgänge verschieden einwirkt. Man kann mit diesem Experiment also weiteres Material für die Diskussion der oben genannten Hypothesen erhalten.

Offen blieb bisher noch, wie diese beiden eben erwähnten Experimente tatsächlich durchgeführt werden können. Es ist nicht möglich, beide Experimente hintereinander mit einer Versuchsperson an demselben Labyrinth durchzuführen, da sich der Lernvorgang des ersten Experimentes auch nach

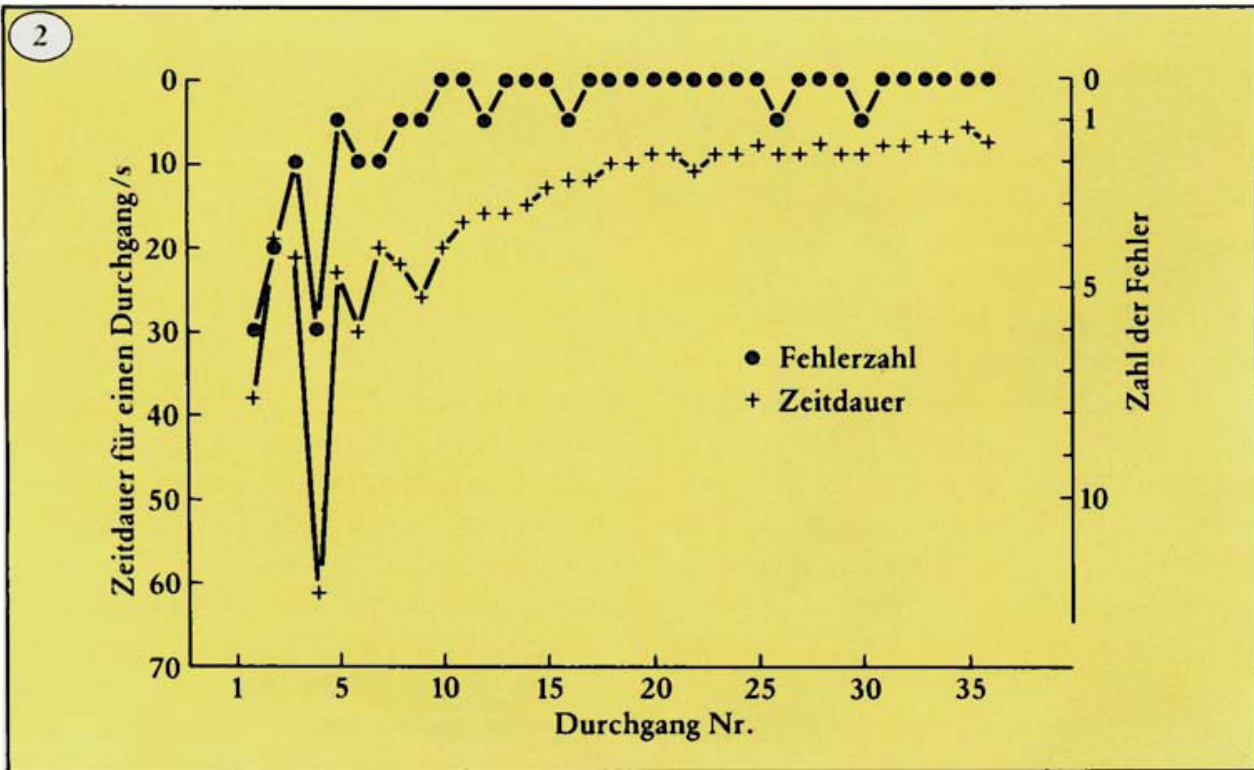




\section{biuz aktuell}

längerer Wartezeit auf den Verlauf der Lernkurve des zweiten Experimentes auswirkt. Man kann den Versuch jedoch so durchführen, daß man entweder zwei verschiedene Versuchspersonen an demselben Labyrinth oder aber eine Versuchsperson an zwei verschiedenen Labyrinthen testet. Aber auch diese beiden Methoden können nicht ohne weiteres angewandt werden. Testet man zwei Versuchspersonen an demselben Labyrinth, so können Unterschiede in den Lernkurven auch durch individuelle Verschiedenheit der beiden Versuchspersonen zustandekommen. Man kann das Problem dadurch umgehen, $\mathrm{da} ß$ man mit Hilfe einer größeren $\mathrm{Zahl}$ von Versuchspersonen die individuellen Unterschiede herausmittelt. Will man dagegen mit einer Versuchsperson zwei verschiedene Labyrinthe testen, so können dabei gleich zwei störende Faktoren auftreten. Einmal kann ein Unterschied in den Lernkurven dadurch zustande kommen, daß beide Labyrinthe verschieden schwierig zu erlernen sind. Außerdem ist es aber möglich, daß die Versuchsperson das zweite Labyrinth stets schneller lernt als das erste, daß sie also sozusagen das Lernen eines Labyrinthes lernt. Die erste Schwierigkeit kann man dadurch umgehen, daß nur solche Labyrinthe für diesen Versuch verwandt werden, bei denen sich in einem Vorversuch ergeben hat, daß beide denselben Schwierigkeitsgrad besitzen. Die zweite Schwierigkeit kann dadurch ausgeschaltet werden, $\mathrm{da}$ die beiden Labyrinthe nicht nur von einer, sondern von zwei Versuchspersonen, aber jeweils in verschiedener zeitlicher Reihenfolge verwandt werden. Man kann dann die vier auf diese Weise erhaltenen „Fehlerkurven“" vergleichen, indem jeweils die zu einem Labyrinth gehörenden zusammengefaßt werden, und dann Unterschiede zwischen diesen Mittelwerten betrachtet werden. Etwa vorhandene Unterschiede sind dann auf einen Einfluß des Zeitdruckes auf den mit der Methode der Fehlermessung erfaßten Lernvorgang zurückzuführen. Dieselbe Uberlegung kann entsprechend für einen Unterschied bei den "Zeitkurven“ angestellt werden. Die in diesem und dem letzten Abschnitt erwähnten Versuche sind sicherlich zu aufwendig, um im normalen Unterricht durchgeführt werden zu können. Ihre Beschreibung ist daher nur als Anregung für eine vertiefende Diskussion dieser Probleme gedacht.

Abschließend soll noch darauf hingewiesen werden, daß ein großer Teil der Experimente, mit denen Fragen zur Funktion des Gedächt- nisses untersucht werden, auf der hier besprochenen Versuchsanordnung beruht. Allerdings wird für Tierexperimente häufig ein sehr einfaches Labyrinth benützt, das nur eine Verzweigungsstelle besitzt (siehe z.B. [1]).

\section{Dr. H. Cruse}

FB Biologie der Universität Kaiserslautern Postfach 3049

D-6750 Kaiserslautern

\section{Literatur}

[1] Domagk, G. F.: Theorien und Experimente zur Gedächtnisspeicherung. Chemie in unserer Zeit 7, 1-8 (1973).

\section{Synthetisches Gen in der Zelle aktiv.}

Auf der Jahrestagung der American Chemical Society, die Ende August in San Francisco stattfand, ist über die erstmalige Totalsynthese eines in der Zelle aktiven Gens berichtet worden. Ein internationales Forscherteam um den Nobelpreisträger Har Gobind Khorana am Massachusettes Institute of Technology, Boston, USA, konnte dieses Ziel nach mehriährigen Arbeiten erreichen, die in ihren ersten Ansätzen schon auf das Jahr 1967 zurückgehen.

Das synthetisierte DNA-Segment, welches für eine Suppressor-transfer-RNA aus E. coli codiert, erstreckt sich über insgesamt 207 Nucleotidpositionen, wovon 199 gepaart sind und jeweils 4 Positionen am Anfang und am Ende zunächst ungepaart bleiben (s. Abbildung). Im Gegensatz zu den früher - 1970 und 1973 - berichteten Gensynthesen derselben Arbeitsgruppe weist der jetzt vorliegende DNA-Doppelstrang auch Kontrollelemente auf, die zur Ablesung innerhalb der Zelle erforderlich sind. So ist dem transkribierten Bereich, welcher mit Position 57 beginnt (s. Abb.), ein Promotor-Bereich (Positionen 6 bis 56) vorgeschaltet, welcher die für den Start der Transkription notwendigen Signalstrukturen enthält. Ein Teil der 21 Nucleotidpaare, welche dem anderen Ende des transkribierten Bereichs folgen (Positionen 183 bis 202), wird als StopSignal für die Transkription angenommen, wenngleich hier die Frage noch offen erscheint, ob eventuell Stopsignale der Phagen-DNA, in welche das künstliche Gen später eingebaut wurde, aushelfen.

Eine wesentliche Voraussetzung für die Synthese des Gens war die Kenntnis seiner Basenpaarsequenz, die weitgehend aus den Basensequenzen der zugehörigen RNAKetten abgeleitet werden konnte, zum Teil aber auch durch direkte DNA-Sequenzierung ermittelt werden mußte. Die Nucleotidsequenz von transfer-RNAE. coli (su') war durch Arbeiten einer Forschergruppe am Labor des Medical Research Council, Cambridge, England, schon 1968 bekannt geworden. Im gleichen Labor wurde später auch die Sequenz des den Positionen 57 bis 97 entsprechenden RNA-Segments ermittelt, welches nur in der Vorläufer-RNA (=primäres Transkript des transfer-RNA Gens) enthalten ist. Aufgrund dieser RNA-Sequenzen ließen sich die Nucleotidsequenzen der Positionen 57 bis 182 des zugehörigen Gens 